\title{
Tennenbaum's Theorem for Models of Arithmetic
}

\author{
Richard Kaye
}

2006-09-23, revised 2006-12-29

\begin{abstract}
This paper discusses Tennenbaum's Theorem in its original context of models of arithmetic, which states that there are no recursive nonstandard models of Peano Arithmetic. We focus on three separate areas: the historical background to the theorem; an understanding of the theorem and its relationship with the Gödel-Rosser Theorem; and extensions of Tennenbaum's theorem to diophantine problems in models of arthmetic, especially problems concerning which diophantine equations have roots in some model of a given theory of arithmetic.
\end{abstract}

\section{Some historical background}

The theorem known as 'Tennenbaum's Theorem' was given by Stanley Tennenbaum in a paper at the April meeting in Monterey, California, 1959, and published as a one-page abstract in the Notices of the American Mathematical Society [30]. It is easily stated as saying that there is no nonstandard recursive model of Peano Arithmetic, and is an attractive and rightly often-quoted result.

This paper celebrates Tennenbaum's Theorem; we state the result fully and give a proof of it and other related results later. This introduction is in the main historical. The goals of the latter parts of this paper are: to set out the connections between Tennenbaum's Theorem for models of arithmetic and the Gödel-Rosser Theorem and recursively inseparable sets; and to investigate stronger versions of Tennenbaum's Theorem and their relationship to some diophantine problems in systems of arithmetic.

Tennenbaum's theorem was discovered in a period of foundational studies, associated particularly with Mostowski, where it still seemed conceivable that useful independence results for arithmetic could be achieved by a 'hands-on' approach to building nonstandard models of arithmetic. Mostowski's own aspirations for the programme are clearly set out in his address to the 8th Congress of Polish mathematicians in September 1953 [17]. Mostowski called for an axiomatic treatment of arithmetic and its models, and cites Ryll-Nardzewski [24] and Rosser and Wang [23] as early proponents of the theory of models of arithmetic. Mostowski places problems of induction and inductive definitions at the forefront of studies in arithmetic, but lists several 'secondary but nevertheless important and interesting problems connected with the axioms of arithmetic of natural numbers': 
What kind of structure have the models of Peano's arithmetic differing from a model composed of natural numbers; in particular, what is their ordinal [i.e., order] type like? After Rosser and Wang [23] we term such models non-standard. Some initial results on these lines have been published by Kemeny [10].

To find out whether by using non-standard models it would be possible to obtain proofs for the independence of classical numbertheoretical problems of the system of arithmetical axioms.

To prove the incompleteness of axiomatic arithmetic without applying the method of arithmetization by giving suitable models showing the consistency and independence of an appropriately chosen number-theoretical axiom.

Mostowski's questions are good ones. Although the order-type of a countable model of PA is easy to determine, it seems that even to this day almost nothing is known about order-types of uncountable models. Nor have any of the classical number-theoretical problems yielded to logical methods of proving independence. Only the last problem, independence results without arithmetization, has yielded results.

Skolem had, of course, already built nonstandard models of arithmetic by an ultrapower-like construction. His 1955 paper [29] is the most accessible and widely quoted, and was written for the proceedings of a symposium in Amsterdam in 1954, but is essentially just a translation of an earlier (1934) paper [28]. Skolem had raised the question of recursiveness of nonstandard models, and Mostowski answered his question, using recursively inseparable r.e. sets to show that no nonstandard model of primitive recursive arithmetic with predicates for all primitive recursive functions can be recursive [18]. Kreisel's review [MR0093483] of this paper is illuminating:

The proof [of Mostowki's main theorem] shows that there is no recursive non-standard model in which all theorems of (quantifierfree) primitive recursive arithmetic are valid. In other words, recursive models are useless for independence problems in full primitive recursive arithmetic. This answers a question raised by Skolem at Amsterdam in 1954 [...]. It is not known if elementary arithmetic with addition and multiplication as the sole non-logical constants has a recursive non-standard model.

Mostowski's theorem was not an isolated event, but followed from a number of earlier papers constructing theories with no recursive models [15] [16], and Kreisel [13] and Putnam [21] had independently obtained similar results to Mostowski's.

As for independence results, Kemeny did produce a model that addressed some questions of independence in 1958 [12]. As one in hindsight would expect, Kemeny's model was shown soon afterwards to fail to satisfy any particularly interesting induction axioms for arithmetic (Gandy [5]). To my mind, the highlight of this period of building recursive models for the purposes of independence results was the results of the early 1960s by Shepherdson, who, using algebraic methods, produced beautiful nonstandard models of quantifier-free arithmetic 
in which he showed number theoretic results such as the infinitude of primes and Fermat's Last Theorem (in fact, for exponent 3) are false [27]. By this time Tennenbaum's theorem was already well known, and Shepherdson and others were certainly aware of the limitations of this approach.

Further results along the same lines as Tennenbaum's appeared soon afterwards. Feferman [3] presented a detailed examination of arithmetization, including the result quoted by Tennenbaum in his abstract that no nonstandard model of full arithmetic can be arithmetically defined. Dana Scott [25] also investigated constructions of models of arithmetic, including an elegant algebraic construction and a proof of Tennenbaum's theorem.

In the following sections, we shall go into more detail, stating and proving Tennenbaum's theorem, and then discussing what theory of arithmetic is actually needed in the model for it to hold. This will bring us on to more recent questions connected with diophantine problems and the solution to Hilbert's 10th problem.

\section{Tennenbaum's Theorem}

I will give a presentation of Tennenbaum's theorem and some variations on it here. All models considered here will be countable models of at least $\mathrm{PA}^{-}$, the theory of the nonnegative part of discretely ordered rings. Except where explicitly stated otherwise, we will regard the standard model $\mathbb{N}$ as an initial segment of each nonstandard model of arithmetic $M$. We shall assume some basic theory from models of arithmetic, such as coding techniques, etc., and to allow us to switch views from recursion theory on $\mathbb{N}$ and recursive sets of formulas we identify a formula with its Gödel-number via some natural Gödel-numbering. All notation not explained here is as in Models of Peano Arithmetic [7].

A structure $M=(M,+, \cdot, 0,1,<)$ is recursive if there is a $1-1$ correspondence $f: \mathbb{N} \rightarrow M$ such that, identifying $\mathbb{N}$ with the domain of $M$ via $f$, the functions ,$+ \cdot$ and relation $<$ on $\mathbb{N}$ are recursive on $\mathbb{N}$. Note that these functions and relation do not need to correspond to the usual ones on $\mathbb{N}$. The structure $M$ is non-recursive if there is no such $1-1$ correspondence. The standard model $(\mathbb{N},+, \cdot, 0,1,<)$ with the usual addition, multiplication and order is recursive, via the identity map $\mathbb{N} \rightarrow \mathbb{N}$. Tennenbaum showed that this is the only such recursive model of arithmetic.

Theorem 2.1 (Tennenbaum, 1959). Let $M=(M,+, \cdot, 0,1,<)$ be a countable model of $P A$, and not isomorphic to the standard model $(\mathbb{N},+, \cdot, 0,1,<)$. Then $M$ is not recursive.

It turns out that the choice of theory here is rather inessential. Indeed Tennenbaum doesn't bother state which theory is taken here, simply writing in his abstract 'provable', which presumably meant 'provable in PA'. The theory PA may be replaced by much weaker sub-theories, including some finitely axiomatized sub-theories. How far one can go in this direction is an interesting question that will be discussed later. 
Tennenbaum's theorem improved on Mostowski's attempts at proving similar results. The key technique was a suitable choice of coding mechanism in arithmetic.

I will present a proof of Tennenbaum's theorem shortly. Before I do so, I would like to indicate at least one aspect of what it says: in some precise sense Tennenbaum's theorem is a model-theoretic version of the Gödel-Rosser incompleteness theorem.

Definition 2.2. For a theory $T$ in the language of arithmetic, denote by $\Pi_{1}-$ $\mathrm{Th}(T)$ the set of $\Pi_{1}$ consequences of $T$, i.e., the set of sentences $\left\{\sigma \in \Pi_{1}: T \vdash \sigma\right\}$. Similarly $\Pi_{n}-\operatorname{Th}(T), \Sigma_{n}-\operatorname{Th}(T)$, etc.

Theorem 2.3 (Rosser). There is no consistent extension $T$ of $P A$ for which $\Pi_{1}-T h(T)$ is recursive.

Proof. I shall sketch a proof assuming Tennenbaum's theorem as stated earlier but for the set of $\Pi_{2}$-consequences of PA, rather than PA itself.

First, assume that $T \supseteq \mathrm{PA}$ is consistent and $\Pi_{1}-\mathrm{Th}(T)$ is recursive. We make a model in the usual Henkin-style using model-theoretic forcing with $\Delta_{0}$ conditions. That is, at stage $k$ of the construction we have a $\Delta_{0}$ condition, i.e., a $\Delta_{0}$ formula $\lambda_{k}\left(w_{0}, \ldots, w_{n_{k}}\right)$ in special 'witnessing' constants $w_{i}$ which is a conjunction of several $\Delta_{0}$ formulas that we want to make true (including all previous conditions in the construction) such that $T+\lambda_{k}\left(w_{0}, \ldots, w_{n_{k}}\right)$ is consistent. The resulting model $M$ will be formed from the $w_{i}$, and by usual techniques we can ensure that $M$ is a $\Sigma_{1}$-elementary submodel of a model $N$ of $T$ together with all the $\lambda_{k}\left(w_{0}, \ldots, w_{n_{k}}\right)$, so in particular $M \vDash \Pi_{2}-\operatorname{Th}(T)$.

The assumption that $\Pi_{1}-\mathrm{Th}(T)$ is recursive allows us to ensure that the whole construction can be carried out recursively. This is because during the construction, we need only decide questions such as, given $\lambda_{k}\left(w_{0}, \ldots w_{n_{k}}\right)$ and a new $\Delta_{0}$ formula $\theta(\bar{w}, \bar{x})$, is $T+\lambda_{k}\left(w_{0}, \ldots, w_{n_{k}}\right)+\theta(\bar{w}, \bar{x})$ consistent? This amounts to asking if

$$
T \vdash \forall w_{0}, \ldots, w_{n_{k}}, \bar{x}\left(\lambda_{k}\left(w_{0}, \ldots, w_{n_{k}}\right) \rightarrow \neg \theta(\bar{w}, \bar{x})\right),
$$

a question that can be effectively decided by looking at $\Pi_{1}-\mathrm{Th}(T)$. Thus the construction is recursive, and indeed the sequence of conditions produced by the construction is also a recursive sequence of $\Delta_{0}$ formulas in the witnessing constants.

This means that the resulting model $M$ is recursive, since it is built from an enumerated set of witnesses $w_{i}$ modulo the recursive equivalence relation $w_{i} \sim$ $w_{j}$ when $w_{i}=w_{j}$ is a conjunct of some condition in the construction. The truth of any $\Sigma_{1}$ sentence $\exists x \theta(x)$ can also be determined: on the one hand by seeing if $\theta\left(w_{i}\right)$ is a conjunct of a condition for some $w_{i}$; and on the other hand by seeing if some other condition $\lambda_{k}\left(w_{0}, \ldots, w_{n_{k}}\right)$ together with $T$ implies $\forall x \neg \theta(x)$. Thus the model $M$ is nonstandard because the truth of $\Sigma_{1}$ sentences in the standard model $\mathbb{N}$ is well-known not to be decidable.

We conclude that if $T \supseteq \mathrm{PA}$ is consistent and $\Pi_{1}-\mathrm{Th}(T)$ is recursive there 
is a recursive nonstandard model of $\Pi_{2}-\mathrm{Th}(T)$, and as $T$ extends $\mathrm{PA}$ this contradicts Tennenbaum's theorem for $\Pi_{2}-\mathrm{Th}(\mathrm{PA})$.

The Gödel-Rosser Theorem is well-known to be related to the following classical result of recursion theory, which we will use to prove Theorem 2.1.

Theorem 2.4. There exist r.e. sets $A, B \subseteq \mathbb{N}$ which are recursively inseparable, i.e., there is no recursive set $C \subseteq \mathbb{N}$ such that $A \subseteq C$ and $B \cap C=\varnothing$.

To prove Theorem 2.1, we will follow Tennenbaum and separate the problem into two subproblems: firstly of saying something about which sets $A \subseteq \mathbb{N}$ are coded in a model $M$, and secondly on the consequences of having nonrecursive sets coded.

Definition 2.5. Let $M$ be a nonstandard model of arithmetic. We define SSy $(M)$, the standard system of sets coded in $M$ to be the set of all $A \subseteq \mathbb{N}$ such that

$$
A=\{n \in \mathbb{N}: M \vDash \eta(n, \bar{a})\}
$$

for some formula $\eta$ and some $\bar{a} \in M$.

In most cases, we may fix a particular formula $\eta$ appropriately and all sets in the standard system appear for this $\eta$ and a suitable choice of parameters $\bar{a}$. By the induction axioms, this will work in PA for any $\eta$ for which the following statement is provable for all pairs of finite disjoint sets $A, B \subseteq \mathbb{N}$ :

$$
\exists \bar{x}\left(\bigwedge_{i \in A} \eta(i, \bar{x}) \wedge \bigwedge_{j \in B} \neg \eta(j, \bar{x})\right)
$$

This happens in the particular case when $\eta(n, x)$ is a first-order formula in the language of PA equivalent to $\exists y\left(p_{n} \cdot y=x\right)$, where $p_{0}=2, p_{1}=3, p_{2}=5$, and so on, enumerating the standard primes. Thus, for nonstandard models $M$ of PA, we have

$$
\operatorname{SSy}(M)=\{A \subseteq \mathbb{N}: \exists a \in M A=\{n \in \mathbb{N}: M \vDash \eta(n, a)\}\}
$$

This formulation of SSy $(M)$ is particularly useful when studying the complexity of addition in a model.

The following lemma is a straightforward application of induction.

Lemma 2.6 (Robinson's overspill lemma). Let $M$ be a nonstandard model of Peano arithmetic, and suppose $\bar{a} \in M$ and $\theta(x, \bar{y})$ is a formula such that $M \vDash \theta(n, \bar{a})$ for each $n \in \mathbb{N}$. Then there is a nonstandard $x \in M$ such that $M \vDash \theta(n, \bar{a})$.

The traditional approach to Tennenbaum's theorem now splits into two parts.

Theorem 2.7. Let $M$ be a nonstandard model of Peano arithmetic. Then SSy $(M)$ contains a nonrecursive set. 
Proof. Let $A, B \subseteq \mathbb{N}$ be r.e., recursively inseparable sets, given by Theorem 2.4. Then these sets are defined (in $\mathbb{N}$ ) by $\Sigma_{1}$ formulas $\exists y \alpha(x, y)$ and $\exists z \beta(x, z)$, respectively, where $\alpha$ and $\beta$ are $\Delta_{0}$. We regard the standard model $\mathbb{N}$ as an initial segment of $M$ and note that $\Sigma_{1}$ formulas are preserved upwards from initial segments to the larger model. So, by this and the disjointness of $A, B$ we have, for each $k \in \mathbb{N}$,

$$
M \vDash \forall x, y, z<k \neg(\alpha(x, y) \wedge \beta(x, z)) .
$$

So by the overspill lemma there is some nonstandard $\zeta \in M$ with

$$
M \vDash \forall x, y, z<\zeta \neg(\alpha(x, y) \wedge \beta(x, z)) .
$$

Now let $C \subseteq \mathbb{N}$ be the set $C=\{n \in \mathbb{N}: M \vDash \exists y<\zeta \alpha(n, y)\}$. By preservation of $\Sigma_{1}$ formulas and the nonstandardness of $\zeta$, we see immediately that $C \supseteq A$, and the above property of $\zeta$ also shows that $C \cap B=\varnothing$. So by our choice of $A$ and $B, C$ is nonrecursive, as required.

Theorem 2.8. Let $M$ be a model of Peano arithmetic for which SSy $(M)$ contains a nonrecursive set. Then $(M,+)$ is not recursive.

Proof. Let $C \in \mathrm{SSy}(M)$ be nonrecursive. Then by remarks made earlier there is $a \in M$ such that

$$
C=\{n \in \mathbb{N}: M \vDash \eta(n, a)\}
$$

for the formula $\eta(n, x)$ which is $\exists y\left(p_{n} y=x\right)$. Then if + in $M$ were recursive so would $C$ be, since on input $n \in \mathbb{N}$ we may compute $p_{n}$ (which is the $n$th prime in $M$ just as it is in $\mathbb{N}$ by preservation between $M$ and $\mathbb{N}$ ) and search for $y \in M$ and $r<p_{n}$ such that $(y+y+\cdots+y)+r=a\left(p_{n} y \mathrm{~s}\right)$. This search is guaranteed to terminate and both $y$ and $r$ are uniquely determined by $n$ and $a$, by Euclidean division in PA. If $r=0$ we conclude $n \in C$, and $n \notin C$ otherwise.

It is natural to ask how far this argument can be pushed, replacing the theory PA by weaker theories. In the form that I have just given it, overspill is only required for $\Delta_{0}$ relations, and the subtheory $I \Delta_{0}$ consisting of some base axioms and induction on $\Delta_{0}$ formulas is strong enough to prove enough facts about Euclidean division, primes (including a formula for the $n$th prime for all standard and sufficiently small nonstandard $n$, though it is still not known if this theory proves the infinitude of primes) for the above argument to go through. This was observed by Cegielski et al [2] and is essentially the proof given in my Models of Peano Arithmetic [7]. Note too that the subtheory mentioned earlier, $\Pi_{2}-\mathrm{Th}(\mathrm{PA})$ of the $\Pi_{2}$ consequences of PA, contains $I \Delta_{0}$. Indeed all axioms of $I \Delta_{0}$ are $\Pi_{1}$ and also axioms of PA; in fact $I \Delta_{0}$ is very much weaker than $\Pi_{2}-\operatorname{Th}(\mathrm{PA})$.

A much sharper result using essentially the same ideas was achieved by Wilmers [31] who showed the same result for the subtheory $I E_{1}$ of $I \Delta_{0}$ where induction axioms are only allowed for bounded existential formulas, i.e., formulas of the form $\exists \bar{y}<p(\bar{x}) q(\bar{x}, \bar{y})=r(\bar{x}, \bar{y})$ where $p, q, r$ are polynomials with nonnegative integer coeficients. Wilmers achieved some improvements on the above argument, firstly by taking $A, B$ to be disjoint r.e., recursively inseparable sets of 
primes, but more particularly by using the MRDP theorem on the diophantine representation of r.e. sets [14]. Interestingly, Wilmers did not ever need to use the provability of the MRDP theorem, just its truth for the standard model $\mathbb{N}$, to get the required sets represented and to achieve the necessary overspill arguments in $I E_{1}$.

Two other methods for showing SSy $(M)$ contains non-recursive sets come to mind. The first is to use a formalization of a (necessarily partial) truth definition in the model $M$. For example, there is a $\Pi_{1}$ formula $\operatorname{Tr}_{1}(x)$ expressing ' $x$ is the Gödel-number of a true $\Pi_{1}$ sentence'. This formula behaves as expected, and in particular is 'correct' on standard formulas, in all models of PA. Then in such a model $M$ the set $C=\left\{\sigma \in \Pi_{1}: M \vDash \operatorname{Tr}_{1}(\sigma)\right\}$ of true standard $\Pi_{1}$ sentences is coded, consistent, and (by the Gödel-Rosser theorem) therefore non-recursive. This method is straightforward and uses well-known results, but because as it relies on a formalization inside the theory of arithmetic, it is not applicable to weaker systems. No such truth definition is known for $I \Delta_{0}$, for instance.

The second method goes back to Scott [26], and uses overspill again. By an overspill argument, $\mathrm{SSy}(M)$ has the property that it is a boolean subalgebra of $P(\mathbb{N})$ closed under relative recursion and König's lemma. (In modern terminology, it is a Scott set.) It follows that every first-order theory (such as PA itself) coded in $M$ (i.e., in SSy $(M)$ ) has a coded complete extension, and of course such sets will not themselves be recursive. (Details are given in Models of Peano Arithmetic [7].) This argument works very well in contexts where overspill is available, and Wilmers [31] shows that $\mathrm{SSy}(M)$ is a Scott set whenever $M \vDash I E_{1}$ is nonstandard. For weaker theories we still seem to need alternative arguments.

In closing this section, I should mention two other strengthenings of Tennenbaum's theorem that have been considered. The first is to start with a nonstandard model $M$ of some weak theory of arithmetic $T$ and find a nonstandard initial segment $I$ of $M$ satisfying PA. Then Tennenbaum's theorem for the initial segment readily implies Tennenbaum's theorem for the original model $M$ by the absoluteness of Euclidean division. For $T=I \Delta_{0}$ a result of this kind was discovered by Cegielski et al $[2]$ and for $T=I E_{1}$ nonstandard initial segments satisfying PA were discovered by Paris [20]. The other strengthening is to consider the reduct of the model $M$ to addition alone. For $M \vDash \mathrm{PA}$, this reduct is a model of Presburger arithmetic and is recursively saturated. Such models are necessarily nonrecursive by similar reasons: Euclidean division gives a notion of standard system, which (by recursive saturation) is a Scott set and therefore contains nonrecursive sets. The same result also holds for nonstandard $M \vDash I \Delta_{0}$ (Cegielski et al [2]) and for nonstandard $M \vDash I E_{1}$ (Wilmers [31]).

\section{Diophantine problems}

In the last section, we saw Tennenbaum's theorem, its relationship with the incompleteness theorems and some subsequent refinements of it down to the theory of bounded existential induction, $I E_{1}$. Some minor improvements were found by the present author by considering the parameter-free induction scheme for 
bounded existential formulas [6], but the theory $I E_{1}$ represents the approximate limit of results of this kind to date. On the other hand Shepherdson [27] found nonstandard recursive models for quantifier-free induction, and Berarducci and Otero have improved this slightly to the case of normal open induction [1]. Between the theories $I E_{1}$ and normal open induction there remains substantial work to be done.

These considerations already put us in the realm of studying diophantine sets, diophantine induction and diophantine definitions, and the issues relating to Hilbert's 10th problem. Wilmers used the MRDP theorem to prove his results about $I E_{1}$. It seems that further progress in the direction of Tennenbaum-type results will require new proofs or at least a more detailed analysis of existing proofs of the the MRDP theorem.

Definition 3.1. We say that a theory $T$ of arithmetic proves the MRDP theorem $\left(T \vdash\right.$ MRDP) if for every $\Sigma_{1}$ formula of the language of arithmetic $\varphi(\bar{x})$ there is an $\exists_{1}$ formula $\psi(\bar{x})$ in the same free variables such that

$$
T \vdash \forall \bar{x}(\varphi(\bar{x}) \leftrightarrow \psi(\bar{x})) .
$$

The definition just given does not make any predetermined choices of equivalent formulas. (In this sense it is not about any first order axiom scheme being provable.) The theory $I \Delta_{0}+\exp$ does prove MRDP (Gaifman and Dimitracopoulos [4]). On the other hand $I \Delta_{0} \vdash$ MRDP is an important open problem related to questions in complexity theory.

Theorem 3.2 (Kaye). Suppose $T$ is a theory of arithmetic extending $\mathrm{PA}^{-}$and $T \vdash M R D P$. Then every nonstandard model of $T$ is nonrecursive.

Proof. Let $A=\{n \in \mathbb{N}: \mathbb{N} \vDash \exists y \alpha(n, y)\}$ and $B=\{n \in \mathbb{N}: \mathbb{N} \vDash \exists z \beta(n, y)\}$ be r.e. recursively inseparable sets, where $\alpha, \beta$ are $\Delta_{0}$. We consider the formula $\theta(w)$ which is

$$
\forall x, y, z<w \neg(\alpha(x, y) \wedge \beta(x, z)) .
$$

The formula $\theta(w)$ is $\Delta_{0}$ and true for all $w \in \mathbb{N}$.

If there is some nonstandard $c \in M$ such that $M \vDash \theta(c)$ then

$$
C=\{n \in \mathbb{N}: M \vDash \exists y<c \alpha(n, y)\}
$$

would be $\Delta_{0}$ definable, contains $A$ ( since $c>\mathbb{N}$ ) and disjoint from $B$. So $C$ would be nonrecursive, but, by considering both the formula defining $C$ and its negation and MRDP in $M$, it is both $\exists_{1}$ - and $\forall_{1}$-definable in $M$, hence is recursive in the operations $+, \cdot,<$ of $M$. We conclude that at least one of these operations must be nonrecursive.

If on the other hand, the standard cut is defined in $M$ by $\theta(w)$, i.e., $\mathbb{N}=$ $\{n \in \mathbb{N}: M \vDash \theta(n)\}$, we take any nonstandard $c \in M$. Then a $\Sigma_{1}$ predicate over naturals $\exists x \psi(n, x)$ is true for $n \in \mathbb{N}$ iff $M \vDash \exists x<c(\theta(x) \wedge \psi(n, x))$. This is a $\Delta_{0}$ formula, so by MRDP in $M$ it is both $\exists_{1}$ and $\forall_{1}$ definable in $M$ so we have shown that every $\Sigma_{1}$ predicate is recursive in the operations $+, \cdot,<$ of $M$, and hence at least one of these operations must be nonrecursive. 
The above result and its proof (which appeared first in Kaye [9]) are interesting, not so much in the result they prove, but in the way that they show that Tennenbaum-type theorems can be proved by means other than overspill, especially by reducing the problem in hand to questions about the standard model. These arguments seem to split into cases: one case mirrors the overspill argument, but if overspill fails then the standard cut is definable in the model in some way which also leads to the required conclusion. Other similar examples will be given shortly.

An old question that has been open since the 1950s (originally raised by Kreisel, I believe) is whether there is an algorithm for deciding provability in the system of arithmetic formulated in the logic without quantifiers. Shepherdson reformulated this question in terms of open induction, and it remains one of the most interesting questions about open induction.

Question 3.3. Is the set $\forall_{1}-T h(I O p e n)$ of universal consequences of open induction recursive?

Another way of stating such a question is to ask if there is an effective method to decide, given a diophantine equation $p(\bar{x})=q(\bar{x})$ where $p, q$ are polynomials with nonnegative integer coefficients, whether there is some model of IOpen containing at least one solution of the equation.

Questions of this type are related to Tennenbaum phenomena, for if $T$ is a theory extending $\mathrm{PA}^{-}$and $\forall_{1}-\mathrm{Th}(T)$ is recursive then by forcing with quantifier-free conditions (as in the proof of Theorem 2.3 above) we may build a recursive model of $\forall_{2}-\mathrm{Th}(T)$. This model is nonstandard because the set of $\exists_{1}$ sentences true in the model can be read off recursively from the construction, but the $\exists_{1}$ theory of the standard model $\mathbb{N}$ is not decidable by the MRDP theorem in $\mathbb{N}$. So Tennenbaum's theorem for $\forall_{2}-\mathrm{Th}(T)$ would imply the undecidability of $\forall_{1}-\operatorname{Th}(T)$.

Now, although it is in fact true that IOpen $=\forall_{2}-$ Th (IOpen), the argument in the last paragraph doesn't seem to help us, as Tennenbaum's theorem fails for IOpen, by Shepherdson's work. In fact, this isn't quite the end of the story as we shall see in a moment, but let us for the moment set our sights a little lower and prove some weaker theorems of the same type. The following result has not previously been published.

Theorem 3.4. The set $\forall \exists^{<}-T h\left(P A^{-}\right)$of consequences of $P A^{-}$of the form $\forall \bar{x} \exists \bar{y}<p(\bar{x}) \theta(\bar{x}, \bar{y})$ with $\theta(\bar{x}, \bar{y})$ quantifier-free is not recursive.

Proof. This is a model theoretic forcing argument with $\forall^{<}$conditions, i.e., conditions of the form $\forall \bar{y}<p(\bar{x}) \theta(\bar{x}, \bar{y})$ with $\theta$ quantifier-free. (Note that up to logical equivalence such formulas are closed under conjunctions.) Our assumption that $\forall \exists<-T h\left(\mathrm{PA}^{-}\right)$is recursive means that we build a recursive model $M$ of $\mathrm{PA}^{-}$with a recursive sequence of conditions. For any tuple $\bar{w} \in M$ of the constructed model, the set of formulas $\exists \forall^{<}-\operatorname{tp}(\bar{w})$ of all $\exists \forall<$ formulas true of the tuple $\bar{w}$ is recursive since given such a formula $\exists \bar{x} \forall \bar{y}<p(\bar{x}) \theta(\bar{w}, \bar{x}, \bar{y})$ we may decide its truth in $M$ by searching simultaenously either for a conjunct of 
a condition of the form $\forall \bar{y}<p(\bar{x}) \theta(\bar{w}, \bar{u}, \bar{y})$ or for a proof that

$$
\mathrm{PA}^{-} \vdash \forall \bar{u}, \bar{v}, \bar{w}(\lambda(\bar{u}, \bar{w}) \rightarrow \neg \forall \bar{y}<p(\bar{v}) \theta(\bar{w}, \bar{v}, \bar{y}))
$$

for some condition $\lambda(\bar{u}, \bar{w})$. In particular, the model $M$ is nonstandard as the set of formulas $\exists \forall<-\operatorname{tp}(0)$ for the standard model $\mathbb{N}$ is non-recursive.

Now take $A, B$ r.e. and recursively inseparable. By the MRDP theorem in $\mathbb{N}$ we may assume $A=\{n \in \mathbb{N}: \exists \bar{y} \alpha(n, \bar{y})\}$ and $B=\{n \in \mathbb{N}: \exists \bar{z} \beta(n, \bar{z})\}$ where $\alpha, \beta$ are quantifier-free. Now consider

$$
\forall x, \bar{y}, \bar{z}<c \neg(\alpha(x, \bar{y}) \wedge \beta(x, \bar{z})) .
$$

If there is no nonstandard $c \in M$ satisfying this, then the standard cut $\mathbb{N}$ is $\forall^{<}$-definable in $M$ and any r.e. set $X \subseteq \mathbb{N}$ would be recursive, since, by MRDP in $\mathbb{N}, X$ is $\{n \in \mathbb{N}: \exists \bar{w} \xi(n, \bar{w})\}$ for some quantifier-free $\xi$, and hence

$$
X=\left\{n \in \mathbb{N}: \exists \bar{w}\left(\bigwedge_{i} \theta\left(w_{i}\right) \wedge \xi(n, \bar{w})\right)\right\}
$$

where $\theta(x)$ is the $\forall^{<}$formula defining $\mathbb{N}$ in $M$. The truth of this can be read off $\exists \forall<-\operatorname{tp}(0)$.

On the other hand, if there is some nonstandard $c \in M$ such that

$$
\forall x, \bar{y}, \bar{z}<c \neg(\alpha(x, \bar{y}) \wedge \beta(x, \bar{z})) .
$$

we consider $C=\{n \in \mathbb{N}: \exists \bar{y}<c \alpha(n, \bar{y})\}$ which contains $A$ and is disjoint from $B$ by choice of $C$, and is also recursive since the truth of $\exists \bar{y}<c \alpha(n, \bar{y})$ can be read off $\exists \forall^{<}-\operatorname{tp}(c)$ for any particular $n$.

We also have (from Kaye [8]),

Theorem 3.5. The set $\forall_{1}-T h\left(I E_{1}\right)$ of $\forall_{1}$ consequences of $I E_{1}$ is not recursive.

As will be clear from the proof of Theorem 3.4, there is still scope for stronger results here. I have given complex conditions by which induction axioms in a theory $T$ may be omitted but nevertheless $\forall_{1}-$ Th $(T)$ be proved nonrecursive by the methods discussed here [8]. Now I shall describe simpler conditions on the theory $T$ for the same result to hold; one still strong enough to prove Theorem 3.5.

To motivate the ideas, connsider first a model $M \vDash I \Delta_{0}$. The theory $I \Delta_{0}$ is strong enough to define exponentiation in a reasonably clean way but cannot prove the statement of the totality of exponentiation, exp: $\forall x \exists y y=2^{x}$. As already mentioned, $I \Delta_{0}+$ exp proves the MRDP theorem. The key application of MRDP in the arguments here is to the formula

$$
\forall x, y, z<w \neg(\alpha(x, y) \wedge \beta(x, z))
$$

where $\alpha, \beta$ are quantifier-free. Let $2_{0}^{x}=x$ and $2_{k+1}^{x}=2^{2_{k}^{x}}$. By the provability of MRDP in $I \Delta_{0}+\exp$ there is some $k \in \mathbb{N}$ (depending possibly on $\alpha, \beta$ ) and quantifier-free $\theta(\bar{u})$ such that $I \Delta_{0}$ proves

$$
\forall w\left(\exists v\left(v=2_{k}^{w}\right) \rightarrow((\forall x, y, z<w \neg(\alpha(x, y) \wedge \beta(x, z))) \leftrightarrow \exists \bar{u} \theta(\bar{u}, w))\right) .
$$


This is by a result for $I \Delta_{0}+\exp$ similar to the well-known theorem of Parikh [19] for $I \Delta_{0}$. Parikh's theorem says that if $I \Delta_{0} \vdash \forall \bar{x} \exists \bar{y} \theta(\bar{x}, \bar{y})$ where $\theta(\bar{x}, \bar{y})$ is $\Delta_{0}$ then the $\bar{y}$ may be bounded by a polynomial in $\bar{x}, I \Delta_{0} \vdash \forall \bar{x} \exists \bar{y}<t(\bar{x}) \theta(\bar{x}, \bar{y})$. The same holds for $I \Delta_{0}+$ exp except the term $t(\bar{x})$ must be a term involving exponentiation, and in fact the axiom exp can be omitted in the conclusion. (This is proved by a model-theoretic argument similar to the one for Parikh's Theorem given for example in Models of Peano Arithmetic, Exercise 6.5 [9].) The particular sentence of interest in our case is

$$
\forall w(\forall x, y, z<w \neg(\alpha(x, y) \wedge \beta(x, z)) \rightarrow \exists \bar{u} \theta(\bar{u}, w))
$$

which is provable in $I \Delta_{0}+\exp$, showing the $\bar{u}$ can be bounded by an exponential term in $w$.

The idea then is to consider the subset $L$ of $M$ consisting of all $w$ for which $M \vDash \exists v\left(v=2_{k}^{w}\right)$. In the case of a nonstandard model of $I \Delta_{0}$, this $L$ will be a nonstandard initial segment, but the important feature is that, by replacing $\exists v\left(v=2_{k}^{w}\right)$ by an appropriate Pell equation, we may take $L$ to be definable by an existential formula.

Definition 3.6. Let $T$ be a theory in the usual language for arithmetic. We say that $T$ virtually proves $M R D P$ if whenever $\varphi(w)$ is $\forall^{<}$then there are $\psi(\bar{v}, w)$ and $\theta(\bar{u}, w)$, both quantifier-free, such that $T \vdash \exists \bar{v} \psi(\bar{v}, n)$ for each $n \in \mathbb{N}$ and

$$
T \vdash \forall w(\exists \bar{v} \psi(\bar{v}, w) \rightarrow(\varphi(w) \leftrightarrow \exists \bar{u} \theta(\bar{u}, w))) .
$$

Theorem 3.7. (a) if $T$ is consistent and virtually proves MRDP then $\forall_{1}-$ $T h(T)$ is nonrecursive. (b) I $E_{1}$ virtually proves $M R D P$.

Proof. (a) Let $A, B$ be r.e. and recursively inseparable and defined by $A=$ $\{n \in \mathbb{N}: \exists \bar{y} \alpha(n, \bar{y})\}$ and $B=\{n \in \mathbb{N}: \exists \bar{z} \beta(n, \bar{z})\}$ as before, and let $\varphi(w)$ be $\forall x, \bar{y}, \bar{z}<w \neg(\alpha(x, \bar{y}) \wedge \beta(x, \bar{z}))$. Take $\psi(\bar{v}, w)$ and $\theta(\bar{u}, w)$ as in the definition and build a recursive model $M$ of $\forall_{2}-\mathrm{Th}(T)$ in which all $\exists_{1}-\operatorname{tp}(\bar{c})$ are resursive, as before. Now choose a nonstandard $c \in M$ such that

$$
M \vDash \exists \bar{u}, \bar{v}(\psi(\bar{v}, c) \wedge \theta(\bar{u}, c)) .
$$

Observe that each $c \in \mathbb{N}$ satisfies the above formula, by properties of $\psi$ and $\theta$ in the definition, so if there were no nonstandard $c \in M$ as above we would conclude that $\mathbb{N}$ is $\exists_{1}$-definable in $M$ and so $\exists_{1}-$ Th $(\mathbb{N})$ can be read off $\exists_{1}-\operatorname{tp}(0)$, which is impossible.

Thus $M \vDash \forall x, \bar{y}, \bar{z}<c \neg(\alpha(x, \bar{y}) \wedge \beta(x, \bar{z}))$ and the set

$$
C=\{n \in \mathbb{N}: \exists \bar{y}<c \alpha(x, \bar{y})\}
$$

is recursive and separates $A, B$.

(b) A Pell equation can be used in place of $y=2^{x}$ to prove an analogous result to

$$
\forall w\left(\exists v\left(v=2_{k}^{w}\right) \rightarrow((\forall x, y, z<w \neg(\alpha(x, y) \wedge \beta(x, z))) \leftrightarrow(\exists \bar{u} \theta(\bar{u}, w)))\right) .
$$

for $I E_{1}$ in place of $I \Delta_{0}$. See Kaye [6] for details. 


\section{References}

[1] Berarducci, Alessandro and Otero, Margarita, A recursive nonstandard model of normal open induction, The Journal of Symbolic Logic, 61, 1996, 1228-1241.

[2] Cegielski, Patrick, McAloon, Kenneth and Wilmers, George, Modèles recursivement saturés de l'addition et de la multiplication des entiers naturels, Logic Colloquium '80 (Prague, 1980), 57-68, North-Holland, 1982.

[3] Feferman, Solomon, Arithmetization of metamathematics in a general setting, Fundamenta Mathematicae, 49, 1960/1961, 35-92.

[4] Gaifman, Haim and Dimitracopoulos, Constantine, Fragments of Peano's arithmetic and the MRDP theorem, Logic and algorithmic (Zurich, 1980), Monograph. Enseign. Math., 30, 187-206, Univ. Genève, Geneva, 1982.

[5] Gandy, Robin O., Note on a paper of Kemeny's, Mathematische Annalen, 136, 1958, 466.

[6] Kaye, Richard, Diophantine induction, Annals of Pure and Applied Logic, 46, 1990, 1-40.

[7] Kaye, Richard, Models of Peano arithmetic, Oxford University Press, 1991.

[8] Kaye, Richard, Hilbert's tenth problem for weak theories of arithmetic, Provability, Interpretability and Arithmetic Symposium (Utrecht, 1991), Annals of Pure and Applied Logic, 61, 1993, 63-73.

[9] Kaye, Richard, Open induction, Tennenbaum phenomena, and complexity theory, Arithmetic, proof theory, and computational complexity (Prague, 1991), Oxford University Press, 1993, 222-237.

[10] Kemeny, John G., Models of logical systems, The Journal of Symbolic Logic, 13, 1948, 16-30.

[11] Kemeny, John G., A note on pseudomodels, The Journal of Symbolic Logic, 17, 1952, 158-159.

[12] Kemeny, John G., Undecidable problems of elementary number theory, Mathematische Annalen, 135, 1958, 160-169.

[13] Kreisel, Georg, Note on arithmetic models for consistent formulae of the predicate calculus II, Actes du XIème Congrès International de Philosophie, Bruxelles, 20-26 Août 1953, vol. XIV, North-Holland, 1953, 39-49.

[14] Matijasevič, Ju. V., The Diophantineness of enumerable sets, Doklady Akademii Nauk SSSR, 191, 1970, 279-282.

[15] Mostowski, Andrzej, On a system of axioms which has no recursively enumerable arithmetic model, Fundamenta Mathematicae, 40, 1953, 56-61.

[16] Mostowski, Andrzej, A formula with no recursively enumerable model, Fundamenta Mathematicae, 42, 1955, 125-140. 
[17] Mostowski, Andrzej, The present state of investigations on the foundations of mathematics (in collaboration with A. Grzegorczyk, S. Jaśkowski, J. Łoś, S. Mazur, H. Rasiowa, and R. Sikorski), Rozprawy Mat., 9, 1955.

[18] Mostowski, Andrzej, On recursive models of formalised arithmetic, Bull. Acad. Polon. Sci. Cl. III, 5, 1957, 705-710.

[19] Parikh, Rohit, Existence and feasibility in arithmetic, The Journal of Symbolic Logic, 36, 1971, 494-508.

[20] Paris, Jeff B., On the structure of models of bounded E $_{1}$-induction, Československá Akademie Věd. Časopis Pro Pěstování Matematiky, 109, 1984, 372-379.

[21] Putnam, Hilary, Arithmetic models for consistent formulae of quantification theory, The Journal of Symbolic Logic, 22, 1957, 110-111.

[22] Rabin, Michael O., Diophantine equations and non-standard models of arithmetic, Logic, Methodology and Philosophy of Science (Proc. 1960 Internat. Congr.), 151-158, Stanford Univ. Press, 1962.

[23] Rosser, J. Barkley and Wang, Hao, Non-standard models for formal logics, The Journal of Symbolic Logic, 15, 1950, 113-129.

[24] Ryll-Nardzewski, C., The role of the axiom of induction in elementary arithmetic, Fundamenta Mathematicae, 39, 1952, 239-263.

[25] Scott, Dana, On constructing models for arithmetic, Infinitistic Methods (Proc. Sympos. Foundations of Math., Warsaw, 1959), 235-255, Pergamon, 1961.

[26] Scott, Dana, Algebras of sets binumerable in complete extensions of arithmetic, Proc. Sympos. Pure Math., Vol. V, 117-121, American Mathematical Society, 1962.

[27] Shepherdson, John C., A non-standard model for a free variable fragment of number theory, Bulletin de l'Académie Polonaise des Sciences. Série des Sciences Mathématiques, Astronomiques et Physiques, 12, 1964, 79-86.

[28] Skolem, Thoralf A., Über die Nichtcharakterisierbarkeit der Zahlenreihe mittels endlich oder abzählbar unendlich vielen Aussagen mit ausschliesslich Zahlvariablen, Fundamenta Mathematicae, 1934, 23, 150-161.

[29] Skolem, Thoralf A., Peano's axioms and models of arithmetic, Mathematical interpretation of formal systems, 1-14, North-Holland Publishing Co., 1955 .

[30] Tennenbaum, Stanley, Non-archimedian models for arithmetic, Notices of the American Mathematical Society, 6, 1959, 270.

[31] Wilmers, George, Bounded existential induction, The Journal of Symbolic Logic, 50, 1985, 72-90. 